\title{
Search for periodic gravitational wave sources with the Explorer detector
}

\author{
P. Astone, ${ }^{1}$ M. Bassan, ${ }^{2}$ P. Bonifazi, ${ }^{3}$ P. Carelli, ${ }^{4}$ E. Coccia,${ }^{2}$ C. Cosmelli, ${ }^{5}$ S. D' Antonio, ${ }^{6}$ V. Fafone,${ }^{6}$ S. Frasca,${ }^{5}$ \\ Y. Minenkov, ${ }^{2}$ I. Modena, ${ }^{2}$ G. Modestino, ${ }^{6}$ A. Moleti, ${ }^{2}$ G. V. Pallottino, ${ }^{5}$ M. A. Papa, ${ }^{7}$ G. Pizzella, ${ }^{2,6}$ L. Quintieri, ${ }^{6}$ F. Ronga, ${ }^{6}$ \\ R. Terenzi, ${ }^{3}$ and M. Visco ${ }^{3}$ \\ ${ }^{1}$ Istituto Nazionale di Fisica Nucleare INFN, Rome, Italy \\ ${ }^{2}$ University of Rome "Tor Vergata" and INFN, Rome, Italy \\ ${ }^{3}$ IFSI-CNR, Roma, Italy \\ ${ }^{4}$ University of L'Aquila, L'Aquila, Italy \\ ${ }^{5}$ University of Rome "La Sapienza" and INFN, Rome, Italy \\ ${ }^{6}$ Laboratori Nazionali di Frascati-INFN, Frascati, Italy \\ ${ }^{7}$ Max Planck Institute of Gravitational Physics, AEI, Golm, Germany
}

(Received 2 February 2001; published 19 December 2001)

\begin{abstract}
We have developed a procedure for the search of signals from periodic sources in the data of gravitational wave detectors. We report here the analysis of one year of data from the resonant detector Explorer, searching for sources located in the Galactic Center (GC). No signals with amplitude greater than $\bar{h}=2.9 \times 10^{-24}$, in the range $921.32-921.38 \mathrm{~Hz}$, were observed using data collected over a time period of 95.7 days, for a source located at $\alpha=17.70 \pm 0.01 \mathrm{~h}$ and $\delta=-29.00 \pm 0.05 \mathrm{deg}$. Our procedure can be extended for any assumed position in the sky and for a more general all-sky search, with the proper frequency correction to account for the spin-down and Doppler effects.
\end{abstract}

DOI: 10.1103/PhysRevD.65.022001

PACS number(s): $04.80 . \mathrm{Nn}$

\section{INTRODUCTION}

Periodic or almost periodic gravitational waves $(\mathrm{GW})$ are emitted by various astrophysical sources. They carry important information on their sources (e.g., spinning neutron stars, accreting neutron stars in binary systems) and also on fundamental physics, since their nature can test the model of general relativity $[1,2]$. The main feature of continuous signals which allows them to be detected is that, despite the weakness of the signal (compared to typical amplitudes for bursts), it is possible to implement procedures that build up the signal to noise ratio (SNR) in time. The natural strategy for searching for monochromatic waves is to look for the most significant peaks in the spectrum. In this case the SNR increases with the observation time $t_{o b s}$. In fact, as $t_{o b s}$ increases, the frequency resolution of the spectrum also increases - the frequency bin gets smaller, $\delta \nu=1 / t_{\text {obs }}$ - thus the noise content in each bin decreases with $t_{o b s}$, while the signal is not dependent on the length of observation time. More specifically, for a periodic signal of amplitude $\bar{h}$ at the frequency $\bar{\nu}$ the squared modulus of the Fourier transform provides $\bar{h}^{2}$ with a noise contribution of $2 S_{h}(\bar{\nu}) \delta \nu$, where $S_{h}(\bar{\nu})$ is the two-sided noise power spectrum of the detector (measured in $\mathrm{Hz}^{-1}$ ). Thus the SNR for periodic signals is:

$$
X_{S N R}=\frac{\bar{h}^{2} t_{o b s}}{2 S_{h}(\bar{\nu})} .
$$

Equation (1) holds if the instantaneous frequency of the continuous signal at the detector is known. The analysis procedure in this case is "coherent," since the phase information contained in the data is used and the sensitivity (in amplitude) increases with the square-root of the time. However in some cases it may be impossible, for various reasons (see later in Sec. III A), to perform a single Fourier transform over all the data. This means that the observation time has to be divided in $M$ sub-periods, such that the spectral resolution of the spectra becomes $\delta \nu^{\prime}=M / t_{o b s}$ and the corresponding $\mathrm{SNR}$ is $M$ times smaller than that given by Eq. (1).

The $M$ spectra can be combined together by incoherent summation, that is by averaging the square modulus. In this case the final spectral resolution is again $\delta \nu^{\prime}$ but there is still some gain as the averaging reduces the variance of the noise in each bin. We obtain

$$
X_{S N R^{\prime}}=\frac{\bar{h}^{2} t_{o b s}}{2 S_{h}(\bar{\nu}) \sqrt{M}} .
$$

In general, if the signal is monochromatic but frequency modulated due to the detector-source relative motion, processing techniques exist which can recover the sinusoidal case if the source direction is known. One of the standard ways of detecting such signals is through appropriate resampling of data, better known in the radio astronomy community (where this technique is commonly used) as "data stretching" (see for example [3]). In the case of radio pulsar searches, the location of the source is usually known (the data come from a radio telescope pointing to a particular direction) but some parameters of the system need to be estimated and this is done by a "timing solution which is phase coherent over the whole data set" [3].

However for gravitational waves, especially when searching a large parameter space, it is doubtful that the strategies developed for radio pulsar searches can simply be adapted: the expected low SNR values for GW signals really modify the nature of the search strategies that can be employed. In recent $\mathrm{ms}$ pulsar searches, for example in [3], the signal is 

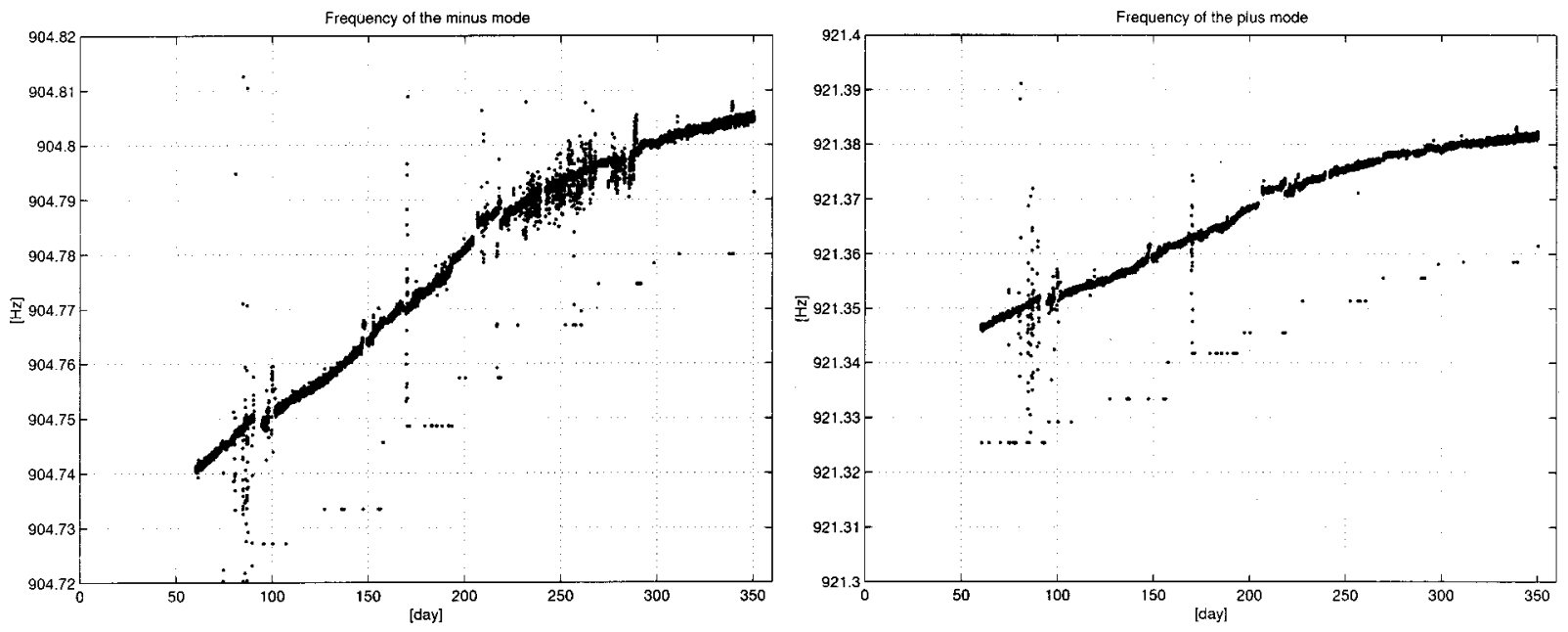

FIG. 1. Left: lower resonance (minus mode) frequency against time (in days of the year). Right: upper resonance (plus mode) frequency against time. The frequency drift observed is due to a slow loss in the electrostatic charge of the transducer.

strong enough to allow suspected pulsars to be identified by visual inspection of the results of the final stages of the analysis procedure.

For gravitational waves the study of the implementation of optimum analysis procedures is still in progress [4-7].

The present paper reports the search for ms periodic sources located in the Galactic Center (GC) assuming their intrinsic frequency to be constant over the analysis time, using the data of a resonant $\mathrm{GW}$ detector.

The procedure we used in this study relies on a data base of fast Fourier transforms (FFTs), computed from short stretches of data (short with reference to the effects of the Doppler shift, as will be described later in this paper). These short FFTs are then properly combined together to provide a new set of FFTs with higher frequency resolution, representing the signal in the frequency range selected for the study. The combination of the elementary FFTs is done using a coherent technique, which provides the SNR given by Eq. (1), and also performs the required Doppler shift corrections.

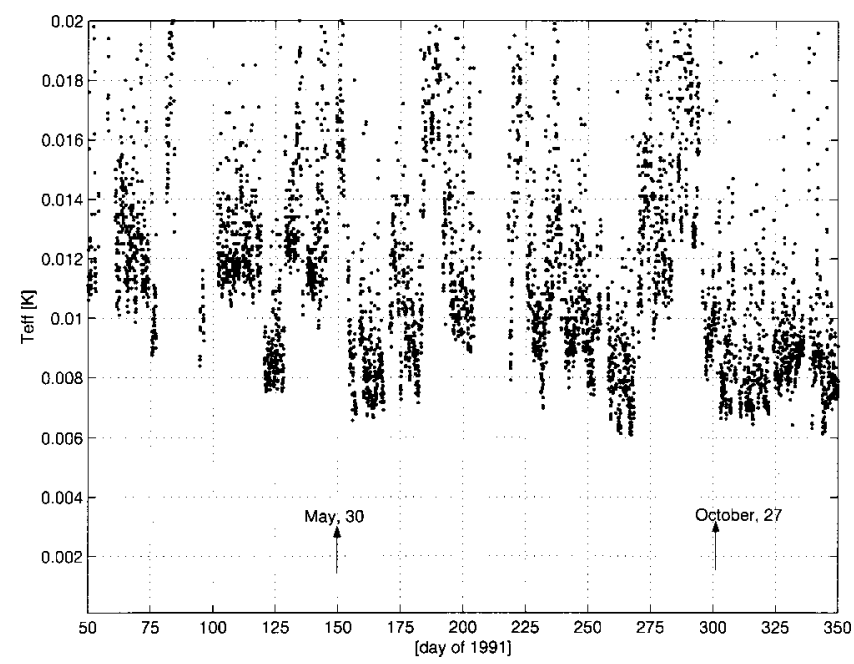

FIG. 2. Hourly averages of the Explorer sensitivity to millisecond bursts, expressed as noise temperature $(\mathrm{K})$ as a function of time.
The paper is organized as follows. In Sec. II we briefly review the characteristics of the detector during the 1991 run; in Sec. III we describe the main aspects of the procedure; in Sec. IV we present the results obtained. The Appendixes clarify some aspects of the analysis procedure and discuss the extent to which the constraints that we have introduced in our procedure can be relaxed in order to account for different sources.

\section{THE EXPLORER DETECTOR}

The Explorer detector is a cryogenic resonant GW antenna located at CERN, at longitude $6^{\circ} 12^{\prime} \mathrm{E}$ and latitude $46^{\circ} 27^{\prime} \mathrm{N}$. The apparatus and the experimental setup of the antenna during the 1991 run have been described in [8] and some results of the data analysis for burst detection are given in $[9-11]$.

The system has two resonance frequencies $\left(\nu_{-}\right.$ $=904.7 \mathrm{~Hz}$ and $\nu_{+}=921.3 \mathrm{~Hz}$ in 1991) where the sensitivity is highest. Figure 1 shows the variations of the two resonance frequencies during the analysis period.

Figure 2 shows the hourly averages of the energy sensitivity $(S N R=1)$ to millisecond bursts, expressed as effective temperature $T_{\text {eff }}$ in kelvin, obtained with an adaptive Wiener filter. ${ }^{1}$ The relation between $T_{\text {eff }}$ and the amplitude of a ms burst is [8] $h=8 \times 10^{-18} \sqrt{T_{\text {eff }}}$ ( $T_{\text {eff }}$ in kelvin).

For periodic waves the sensitivity of a bar detector at its resonances is given by $[13,14]$ :

$$
\bar{h}=2.04 \times 10^{-25} \sqrt{\frac{T}{0.05 \mathrm{~K}} \frac{2300 \mathrm{~kg}}{M} \frac{10^{7}}{Q} \frac{900 \mathrm{~Hz}}{\nu_{0}} \frac{1 \mathrm{day}}{t_{\text {obs }}}}
$$

where $T$ is the bar temperature, $M$ its mass, $Q$ the merit factor, $\nu_{0}$ the resonance frequency of the mode and $t_{\text {obs }}$ the

\footnotetext{
${ }^{1}$ The sensitivity obtained with a matched filter was, on average, better by a factor of 2 . The comparison between the two filtering procedures is shown in [12].
} 
observation time. After one year of effective observation, the minimum detectable $\bar{h}$ (amplitude detectable with $X_{\mathrm{SNR}}$ $=1$ ), using the nominal parameters of the Explorer detector $\left(T=2 \mathrm{~K}, M=2300 \mathrm{~kg}, Q=10^{6}\right)$, is

$$
\bar{h}=2 \times 10^{-25}
$$

in a bandwidth of $\simeq 2 \mathrm{~Hz}$ around the two resonance frequencies and

$$
\bar{h} \simeq 2 \times 10^{-24}
$$

in a bandwidth of $16 \mathrm{~Hz}$ between the two resonances. For the NAUTILUS [15] or AURIGA [16] detectors (with $T$ $=0.1 \mathrm{~K}, Q=10^{7}$ ) we get a value $\bar{h} \simeq 1.5 \times 10^{-26}$ at the resonances.

\section{MAIN FEATURES OF THE ANALYSIS PROCEDURE}

In the search for continuous signals there are a number of issues that need to be kept in mind regarding the signals that might be present, the apparatus and the quality of the data. As far as the source is concerned, it is not possible to set up a single procedure capable of searching over all types of periodic signals. In this analysis, we concentrated on periodic signals such as those expected from isolated neutron stars with weak spin down, i.e. we ignored the spin down parameters. ${ }^{2}$ Moreover we did not consider the effects of proper accelerations of the source. Thus our model assumes that the frequency behavior of the signal exclusively depends on the Doppler effect caused by the motion of our Earthbased detector relative to the source location. Let us quote some basic figures:

The Doppler effect has two periodic components (see Appendix A for details). The first one, due to the revolution motion of the Earth over a period of 1 year produces a maximum time derivative of the frequency given by

$$
b=\left|\frac{d \nu_{\text {orb }}}{d t}\right|_{\max }=\nu_{0} 1.98 \times 10^{-11} \mathrm{~Hz} / \mathrm{s}
$$

where $\nu_{0}$, measured in $\mathrm{Hz}$, is the intrinsic frequency of the source.

The second one, due to the rotation of the Earth over a period of 1 sidereal day, produces a maximum time derivative of the frequency given by

$$
a=\left|\frac{d \nu_{\text {rot }}}{d t}\right|_{\max }=\nu_{0} 11.24 \times 10^{-11} \cos \phi \mathrm{Hz} / \mathrm{s}
$$

where $\phi$ is the latitude of the detector and $\nu_{0}$ is measured in $\mathrm{Hz}$.

The observation is also affected by modulation in the amplitude. This is due to the varying orientation of the detector

\footnotetext{
${ }^{2}$ However it is possible, using our procedure, to take into account the spin down. This will be the next step in the development of the procedure.
}
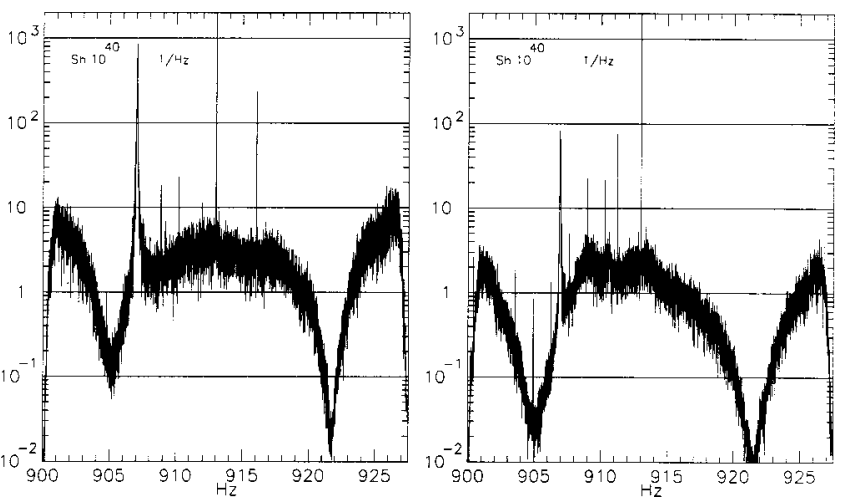

FIG. 3. The figure shows two power spectra of the detector, both obtained during periods of "good" operation of the apparatus: during November (left) and September (right) 1991. The $y$-axis is $S_{h}$ $\times 10^{40}$ in units of $1 / \mathrm{Hz}$. The $x$-axis is the frequency in [Hz]. Comparing the two power spectra, it is easy to see the nonstationarity of the system

with respect to the source because of the Earth's motion. It may also be a consequence of the polarization of the wave.

As shown, for example, in [17], this modulation spreads the signal power across side bands, spaced at $1 / 24$ hours. The amplitude modulation observed using a resonant bar detector is given by the geometrical part of the detector cross section [18]:

$$
\Sigma=\Sigma_{0} \cdot \Phi\left(\theta, \epsilon_{p}, \phi_{p}\right)=\Sigma_{0} \sin ^{4} \theta\left(\frac{1-\epsilon_{p}}{2}+\epsilon_{p} \cos ^{2}\left(2 \phi_{p}\right)\right)
$$

where $\epsilon_{p}$ is the degree of the wave linear polarization $\left(\epsilon_{p}\right.$ $=1$ when there is linear polarization, $\epsilon_{p}=0$ when there is no polarization),

$$
\Sigma_{0}=\frac{16}{\pi}\left(\frac{v}{c}\right)^{2} \frac{G}{c}
$$

is the (two-sided) mechanical part of the cross section ( $M$ =bar mass, $v=$ sound velocity in the bar), $\theta$ is the angle between the bar axis and the wave direction of propagation, $\phi_{p}$ is the angle between the bar axis and the wave polarization plane. The cross section is maximum when the parameters are $\theta=\pi / 2, \phi_{p}=0, \epsilon_{p}=1$. If the source location and the polarization state are known, it is possible to demodulate the amplitude of the observed signal.

A major consideration in developing the analysis is that the operation of the detector is not continuous and the noise is not stationary. An example of this is given in Fig. 3 which shows two power spectra, each computed over two hours of data, in November and September 1991. There are several lines from periodic disturbances which are not stationary, and the noise level differs between the two spectra.

\section{A. The length of the FFTs in the data base}

Our frequency domain data base consists of "elementary spectra," each obtained by performing the FFT (fast Fourier transform) of a given number of samples of the data, over a 
duration $t_{0}$, recorded by our detector. The signal sensitivity of each spectrum, according to Eq. (1), depends on the duration $t_{0}$. As our observations are affected by the Doppler shift, we have chosen the duration $t_{0}$ of the "elementary spectra" to be the longest possible compatible with the requirement that the signal should "appear as monochromatic" during $t_{0}$. Clearly some assumptions about the frequency variation must be made. In principle, in order to achieve a higher SNR in the short spectrum, some preprocessing could take place by setting a coarse grid on the parameter space (i.e., the part of the sky that is being investigated) and performing suitable data stretching for each point in that parameter space. In this way a signal coming from that parameter space would appear as monochromatic in the resulting spectrum. As a consequence the size of the data base is increased by a factor equal to the number of points in parameter space, but there is a gain in SNR because of the higher spectral resolution.

As stated above, we restricted our analysis to the case where the only frequency changes are due to the Doppler effect of the detector motion relative to the source.

As shown in Appendix A, the time duration $t_{0}$ must be

$$
t_{0}<\frac{1}{\sqrt{a+b}}=8.7 \times 10^{4} / \sqrt{\nu_{0}} \mathrm{~s},
$$

where $\nu_{0}$, measured in $\mathrm{Hz}$, is the source intrinsic frequency, $a$ and $b$ are given in Eq. (6) and Eq. (5) and we have put $\cos \phi=1$ (maximum possible value). In the particular case of Explorer we get $t_{0}<10 \times 10^{4} / \sqrt{\nu_{0}} \mathrm{~s} \quad(\phi=46 \mathrm{deg})$.

Thus, to construct the elementary spectra of our data base, we choose a duration of $t_{0}=2382.4 \mathrm{~s}=39.7$ minutes, corresponding to $2 N=131072$ samples, recorded with sampling time of $18.176 \mathrm{~ms}$. With this choice of $t_{0}$, as shown in Appendix A, the maximum Doppler frequency variation (for Explorer at $\nu_{0}=921.38 \mathrm{~Hz}$ ) during $t_{0}$ is $0.215 \mathrm{mHz}$, smaller than the resulting frequency bin $\delta \nu=0.419 \mathrm{mHz}$.

\section{B. The FFT header}

The header of each elementary spectrum of the database contains various information about the original data. This allows stretches of data that are noisier than others to be vetoed or weighted differently and thus best exploits the potential of the data.

Some of the information contained in the header relates to the data structure, some of it to the operational status of the detector and some to data quality. For example, the date and time of the first sample of the data series that the FFT is computed from is stored, along with the frequency resolution of the FFT and the type of time-domain windowing used. There are also system parameters that vary in time: the frequencies of the two modes and of the calibration signal, the level of Brownian noise and the merit factors of the two modes, the wide band noise level and the status of the operation flags (normal operation, maintenance works, liquid helium refilling). Some of this information was used to set a threshold for vetoing the data.

\section{The procedure for combining the spectra coherently}

For the targeted search described here the basic FFTs are combined coherently to improve the final sensitivity. The following is an outline of how this is done (details are given in Appendix B).

(i) Take an FFT over a bandwidth $B$ including the resonances of the detector. Let $\delta \nu$ be the frequency resolution and $2 N$ the number of data samples.

(ii) Take the data from $n^{\prime}$ bins in the frequency range $\Delta \nu$ of the actual search; $n^{\prime}=N \Delta \nu / B$.

(iii) Build a complex vector that has the following structure: (a) the first datum equal to zero; (b) the next $n^{\prime}$ data equal to those from the selected bins of the FFT; (c) zeros from bins $n^{\prime}+1$ up to the nearest subsequent bin numbered with a power of 2 (let us say that this way we have $n$ bins); (d) zeros in the next $n$ bins. So, we end up with a vector that is $2 n$ long.

(iv) Take the inverse FFT of the vector. This is a complex time series that is the "analytical signal" representation of the signal in the band $\Delta \nu$. It is shifted towards lower frequencies and it is sampled at a sampling rate lower by a factor $2 N / 2 n$ compared to the original time data. ${ }^{3}$ The time of the first sample here is exactly the same as the first datum used for the data base and the total duration is also that of the original time stretch. There are fewer data because here the sampling time is longer.

(v) Repeat the steps outlined above for all the $R$ FFTs;

(vi) If they all come from contiguous time stretches simply append them one after the other in chronological order. If they are not all contiguous set to zero those stretches where data are missing.

(vii) To correct for the Doppler effect ${ }^{4}$ from sources from a given direction, multiply each sample of the sequence by

$$
\exp ^{-j \phi\left(t_{i}\right)} \text {. }
$$

$t_{i}$ are the times of the samples and $\phi\left(t_{i}\right)=\int_{t_{s}}^{t_{i}} \Delta \omega_{D}(t) d t$.

$\Delta \omega_{D}(t)$ is the Doppler correction, in angular frequency, at the time $t$ of the $i$ th sample: $\Delta \omega_{D}(t)=\omega_{D}(t)-\omega_{s}$, where $\omega_{D}(t)$ is the frequency observed at the detector, due to the Doppler effect from a given source that emits at a constant frequency $\omega_{s}, t_{s}$ is the start time of the overall FFT being constructed.

We note that the frequency correction is performed on the sub-sampled data set, and this is one of the advantages of the procedure.

(viii) Perform the FFT of the $2 n \cdot R$ data thus obtained.

(ix) Finally, take the squared modulus of the FFT thus obtained. This is the power spectrum of the original time series, in the frequency range $\Delta \nu$, with the full spectral reso-

\footnotetext{
${ }^{3}$ The construction of the analytical signal is a standard procedure of low-pass filtering for a bandpass process. In fact the analytic signal is zero on the left frequency plane, thus avoiding aliasing effects in the low-pass sampling operation [19].

${ }^{4} \mathrm{We}$ could also take into account other causes of frequency shifts such as those affecting the intrinsic frequency of the source.
} 
lution $\delta \nu / R$, and, more importantly, with the full sensitivity given by Eq. (1).

A signal exhibiting frequency variability smaller than the variability we have corrected for, should appear wholly within a single frequency bin, and its resulting SNR will be that of Eq. (1).

\section{An example of the procedure for combining spectra}

The procedure was tested on simulated signals added to the data. We shall now briefly review the results of these tests. Such simulations, although simple in principle, present practical design problems which demand extreme care in the implementation. The simulated signal is constructed in the time domain and then it is handled in exactly the same way as the real detector data (details on the data handling procedures are given in the Appendixes). Each FFT of the signal is then added to the corresponding FFT in the data base.

$$
s(n \Delta t)=\bar{h}(n \Delta t) \sin \left[\phi(n \Delta t)+\phi_{0}\right]
$$

where $\Delta t$ is the sampling time, $n=0,1 \ldots 131072, \phi_{0}$ the initial phase,

$$
\phi(n \Delta t)=\int_{0}^{n \Delta t} \omega_{D}(t) d t
$$

where $\omega_{D}(t)$ is the frequency at the detector due to the Doppler shift at time $t$. Using the discrete form of Eq. (11) we may write the phase at time $t_{i}$ :

$$
\phi_{i}=\phi_{i-1}+\omega_{D i} \Delta t .
$$

We report here an example of the results of a simulation performed in the absence of noise (we set the detector FFTs to zero, before adding them to the simulated signal).

Figure 4 shows the comparison of the two power spectra obtained from a source assumed to be in the GC, emitting at $921.3 \mathrm{~Hz}$, before and after Doppler removal. It is clear that the spread and the shift in the signal frequency (top figure) have been properly corrected (bottom figure). Here the observation time is 36 hours and the frequency resolution is $6.4 \ldots \mu \mathrm{Hz}$.

The level of the signal, after Doppler removal, is that which would be expected $(\bar{h}=1.0 / \sqrt{\mathrm{Hz}})$. An accurate analysis of the residual error after Doppler removal (this error is defined as the instantaneous difference between the time signal after correction and the time signal in the absence of modulation) showed that this residual error was always less than $0.7 \%$.

\section{THE ANALYSIS OF THE EXPLORER DATA}

We analyzed data taken during the period between March and December 1991. These data sets comprise 4954 FFTs from March to July and 4384 from August to December. After a preliminary analysis of the features of the spectra, with particular reference to their sensitivity performance, we decided to veto the spectra with Brownian noise larger than $7.8 \mathrm{~K}$ (i.e., three times greater than the expected value of 2.6
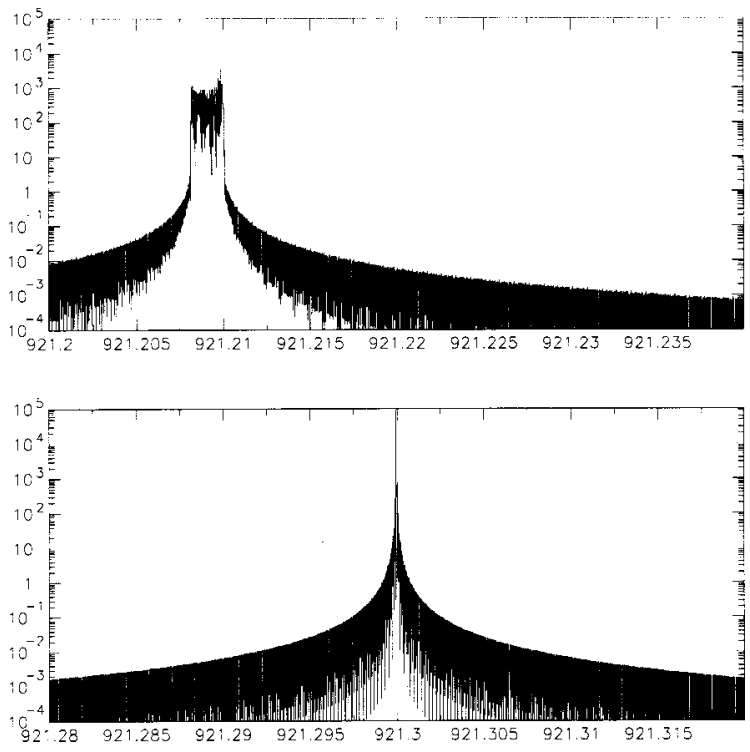

FIG. 4. Simulation of a signal (at $921.3 \mathrm{~Hz}$ ) from the GC, over 36 hours of data. Top: Spectrum of the simulated data. Bottom: Spectrum after Doppler removal. The $y$-axis is the spectrum $\times 10^{40}$, in units of $1 / \mathrm{Hz}$. The $x$-axis is the frequency in $\mathrm{Hz}$.

$\mathrm{K}$ [8]). With this criterion we vetoed 807 spectra, that is $\simeq 10 \%$ of the total.

A comment on the accuracy of the timing of the data is necessary at this point: the absolute time recording had an indetermination of the order of $10-20 \mathrm{~ms}$ [8] at the beginning of each new run. This was due to the fact that, although the time was checked against the Swiss time signal HBG with an accuracy of a few $\mathrm{ms}$, the software procedure at the start of each run introduced an imprecision of $\simeq 10-20 \mathrm{~ms}$.

On the other hand we are confident about the precision of the rubidium clock, which was used to determine the sampling time. As a consequence we could combine coherently only data obtained from one single acquisition run.

The strategy for the analysis procedure was thus the following: (i) choose the frequency bandwidths to be analyzed, and calculate new-higher resolution-FFTs on each new run, for each of these chosen bandwidths; (ii) choose the coordinates of the source direction and correct for Doppler effect and for amplitude modulation, using the procedure described in Sec. III. We ignored possible polarization of the waves.

This analysis was focused on possible sources in the GC, at $\alpha=17.7 \mathrm{~h}, \delta=-29.0 \mathrm{deg}$.

To calculate the Doppler shift we used the JPL ephemerides (JPLEPH.405) and software routines from the U.S. Naval Observatory (NOVAS). The amplitude modulation was removed from the data by multiplying the data by the factor $\sin ^{4} \theta(t)$ (in other words, the data were weighted on the basis of the source-detector direction).

In 1991 we collected data over 51 separate runs and therefore, applying the procedure outlined above, we obtained 51 separate FFTs. Each one has a different frequency resolution, according to its length in time. The analysis of the 51 FFTs could only be done by combining their information "inco- 


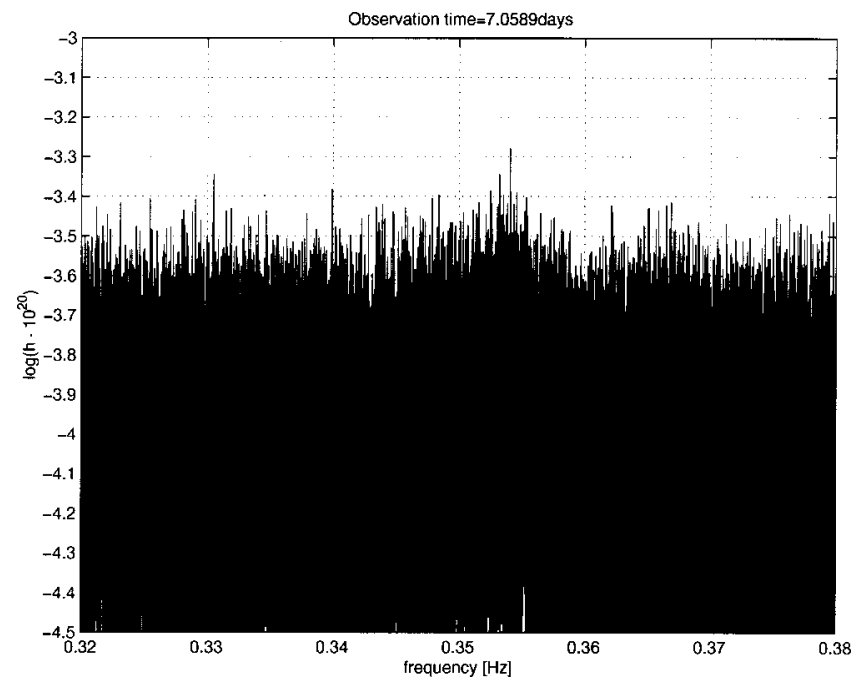

FIG. 5. Amplitude $\bar{h}$ of signals detectable with $\mathrm{SNR}=1$, over 7.05 days (from June, 8th), in the range 921.32-921.38 Hz. The data have been corrected to look for signals from the GC. The $x$-axis is the frequency in units of $\mathrm{Hz}$, having subtracted $921 \mathrm{~Hz}$.

herently," thus combining the spectra for example by incoherent summation. Obviously, this reduced the sensitivity of the final analysis [see Eq. (2)].

We report in this paper the results of the analysis of the data around the frequency of the plus mode (the mode in Fig. 1 , bottom).

\section{A. The analysis of 95.7 days of data}

First of all we give an example using data over one week in June 1991. Figure 5 shows one spectrum, obtained over $t_{\text {obs }}=7.05$ days from day 159.8 (June, 8th) in the bandwidth 921.32-921.38 Hz. The figure is normalized in terms of the amplitude $\bar{h}$ that would give $X_{S N R}=1$ for sources in the GC.

The level of the noise is $(1.2 \pm 0.7) \times 10^{-24}$, in good agreement with that expected for Explorer [using Eq. (3), with $T=2.6 \mathrm{~K}$ and $Q=10^{6}$, we get $1.9 \times 10^{-24}$ ].

The Doppler correction needed for signals from the GC was applied to the data. The highest peak found is $\bar{h}=5.2$ $\times 10^{-24}$.

For comparison we show in Fig. 6 the case where no Doppler correction was applied. It is possible to note a high peak $\left(\bar{h}=1.2 \times 10^{-23}\right)$, which disappears when the Doppler correction is applied, as it spreads this contribution over several frequency bins. It is most likely that the peak in Fig. 6 was due to the apparatus.

We started the analysis using only the data from three consecutive runs in May and June. We averaged the corresponding spectra over a total observation time of $t_{o b s}$ $=21.177$ days from day 128.53 (May 8th). The analysis was done in the frequency range $921.32-921.38 \mathrm{~Hz}$, where the antenna noise spectrum was flat, as shown in Fig. 7, with $\bar{h}$ level $(1.6 \pm 0.5) \times 10^{-24}$. We notice that the average level for the 21.177-day period is roughly the same as that for the 7.05-day period, and the standard deviation decreases, as it should do, by a factor of the order of $\sqrt{21.177 / 7.05} \simeq \sqrt{3}$ (the

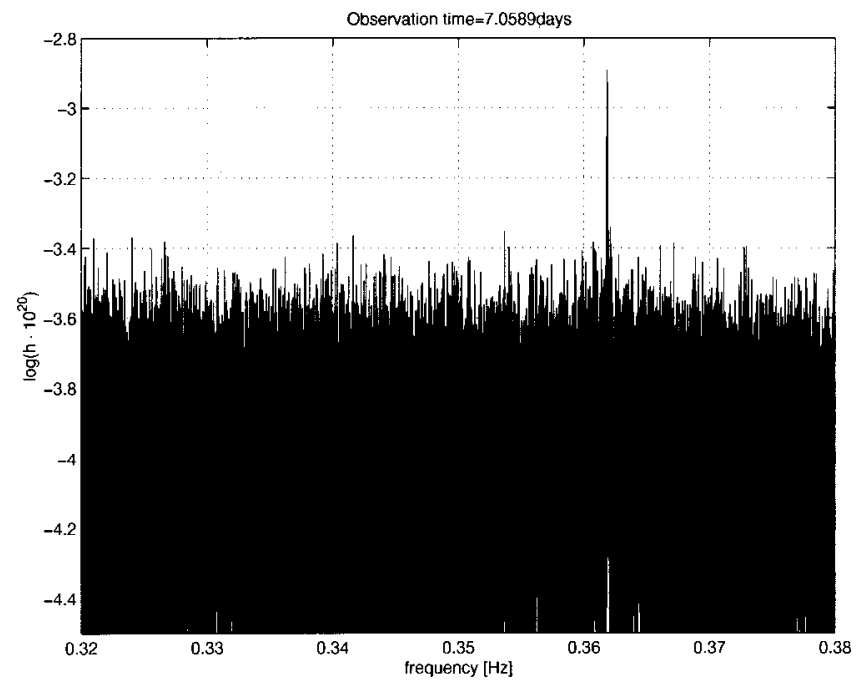

FIG. 6. The same data as for Fig. 5 but in this case no Doppler correction is applied. The high peak disappears when applying the Doppler correction, thus it is not due to monochromatic signals from the GC.

small difference is due to the nonstationarities during the three time periods).

No spectral lines were detected with amplitude (at the detector) greater than $\bar{h}=4.1 \times 10^{-24}$ during this period.

To set an upper limit on the amplitude of possible signals from GC in the chosen bandwidth, we decided to check the efficiency of detection, given the noise of the detector. We therefore added signals with different amplitudes and phases to the data, using data without Doppler correction, since the efficiency of detection, on the average, does not depend on the Doppler effect.

We added four different families of signals, each family consisting of 20 sinusoids with the same amplitude but different phases.

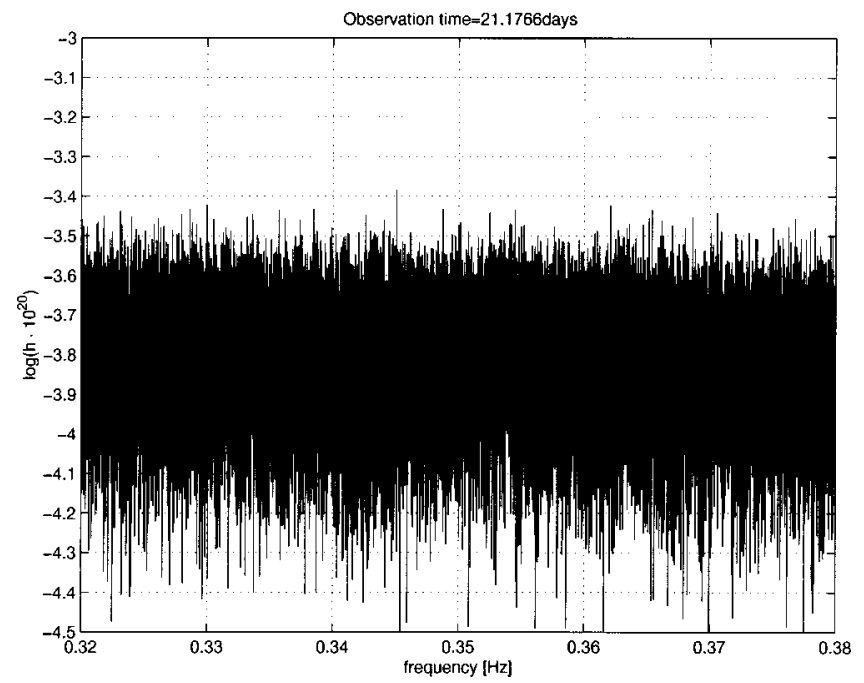

FIG. 7. Average $\bar{h}$ from GC obtained averaging the spectra of 3 runs, 7 days each, from May, 8th. The $x$-axis is the frequency, in Hz-921 Hz. 


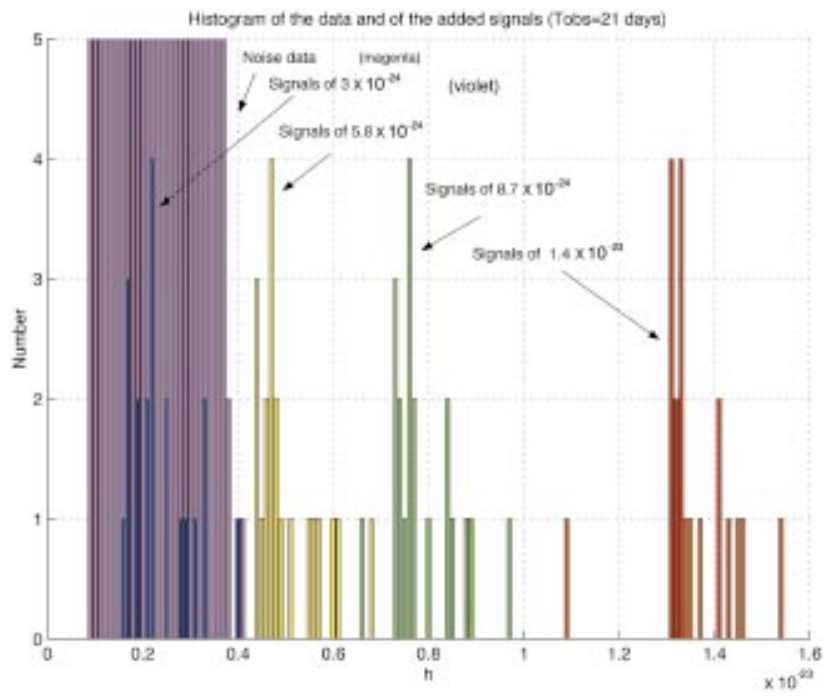

FIG. 8. (Color) Histograms in the range 921.32-921.38 Hz. $t_{o b s}=21$ days. The histograms report the data (magenta) and the four different families of simulated signals (violet, yellow, green, red). For clarity, the $y$-axis numbers above five have not been plotted. The $x$-axis ranges from $1.0 \times 10^{-24}$ to $1.6 \times 10^{-23}$, with step $0.1 \times 10^{-24}$. The histograms show clearly that the efficiency of detection is 1 for the simulated signals corresponding to the yellow, green and red plots.

The results of the analysis are shown in Fig. 8. The histograms report the detected amplitudes for the four different families of simulated signals. On the $x$-axis we have values ranging from $\bar{h}=1.0 \times 10^{-24}$ to $1.6 \times 10^{-23}$, with an interval of $0.1 \times 10^{-24}$.

The nominal amplitudes of the added signals are 1.4 $\times 10^{-23}, 8.7 \times 10^{-24}, 5.8 \times 10^{-24}$, and $3.0 \times 10^{-24}$. If we consider only the three families of higher signals, the histograms show very clearly that all these signals have been well detected. Even the smallest of these 60 signals is well above

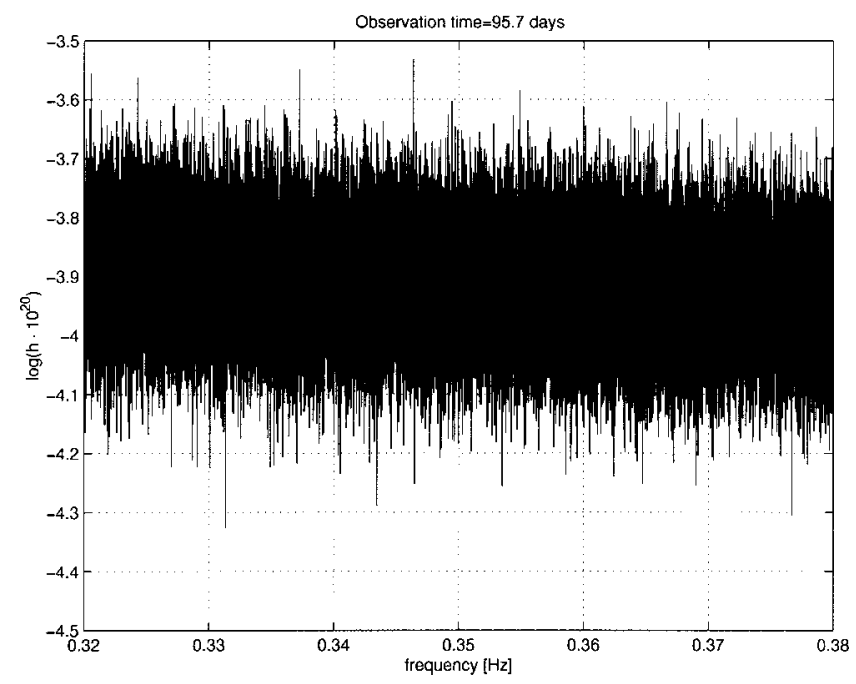

FIG. 9. Average $\bar{h}$ from GC obtained using data from May to December (95.7 days) in the bandwidth 921.32-921.38 Hz. The $x$-axis is the frequency, in Hz-921 Hz. the standard deviation of the data (the smallest three signals have $\bar{h}=4.4 \times 10^{-24}$, which is roughly 10 times the noise standard deviation). Thus, for these signals, on a time basis of only 21 days, the efficiency of detection is 1 . On the contrary, the histograms show that the efficiency of detection for the signals at the lowest level $\left(3.0 \times 10^{-24}\right)$ is very poor.

Thus, on the basis of 21 days of data, we exclude the possibility that, in the GC, there are sources having a spindown age $\tau \geqslant 3 \times 10^{7}$ years emitting signals with frequency in the range 921.32-921.38 Hz and strength (on Earth) greater than or equal to $\bar{h}=5.8 \times 10^{-24}$.

We now consider the eleven longest runs between May and December, with observation times ranging from 7.7 to 12.8 days, giving a total effective observation time of 95.72 days. We averaged these spectra, after adding the necessary zeros to obtain the same virtual resolution (this produces a change in the SNR and therefore a re-calibration of the spectra is needed).

The start times of these eleven runs, are days 128.53, 137.29, 159.82, 171.43, 213.94, 225.32, 301.61, 312.37,
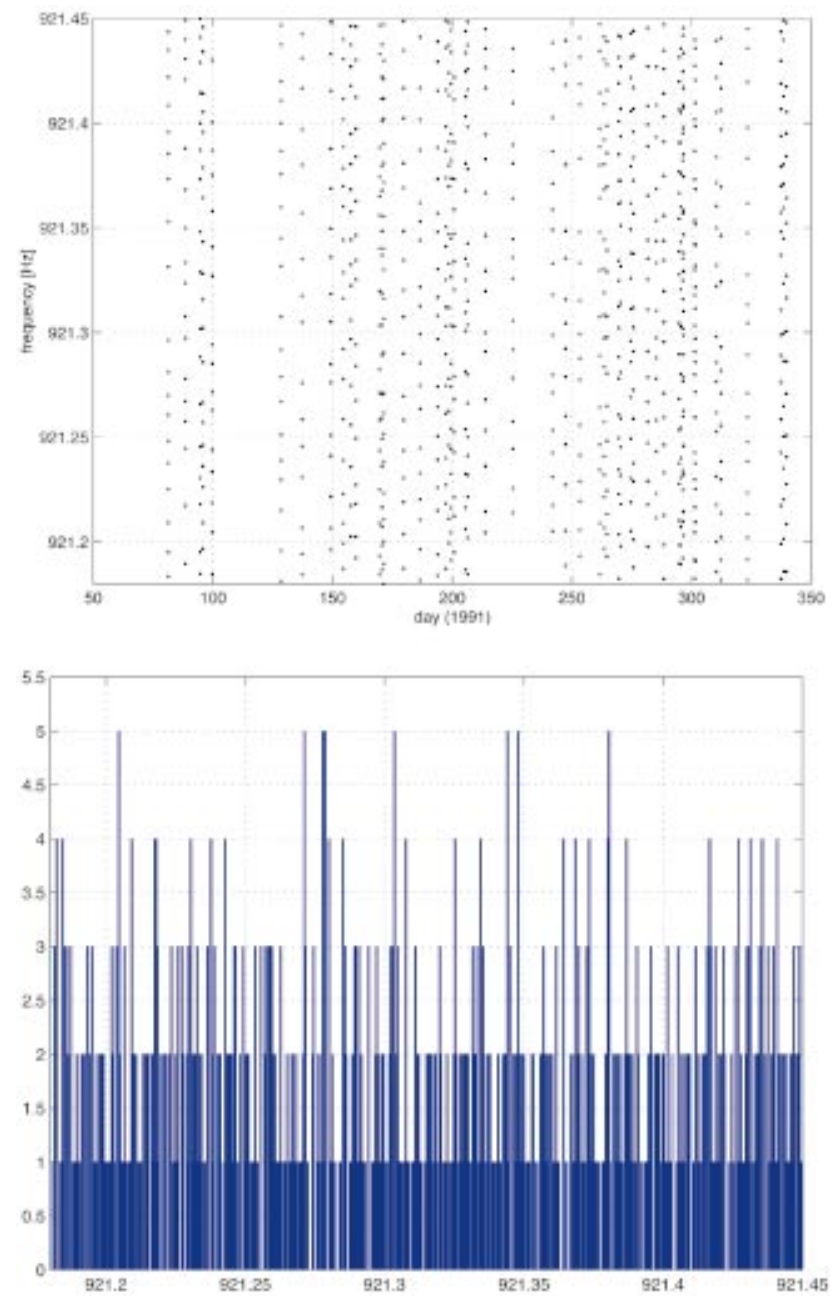

FIG. 10. (Color) The incoherent analysis over 51 spectra from May to December. The upper plot shows the time-frequency behavior of the peaks with $X_{S N R} \geqslant 4$, in each spectrum. The lower plot is their histogram versus frequency. 
323.46, 339.68. Figure 9 shows the average $\bar{h}$ for the frequency range 921.32-921.38. In the frequency bandwidth $921.32-921.38 \mathrm{~Hz}$ over these 95.7 days the noise level is $(1.2 \pm 0.2) \times 10^{-24}$, well in agreement with the expected value. No lines with amplitude greater than $\bar{h}=2.9 \times 10^{-24}$ are apparent.

The standard deviation is a factor 2.5 lower than the standard deviation obtained using only 21 days, thus we expect the efficiency of detection over the 95 days to be of the order of unity even for signals of $\bar{h}=(2-3) \times 10^{-24}$.

Thus, we exclude the possibility that, in the GC, there are sources having a spindown age $\tau \geqslant 10^{8}$ years, emitting signals with frequency in the range $921.32-921.38 \mathrm{~Hz}$ and strength (on Earth) greater than or equal to $\bar{h}=2.9 \times 10^{-24}$.

\section{B. A first attempt to incoherent analysis by frequency tracking}

The analyzed period consists of 51 runs, leading to 51 spectra of different resolution. It is not convenient to average these spectra as done before for the eleven longest ones, because now their durations are very different one from each other. They can be analyzed using other methods-for example, by looking for patterns in the time evolution of their spectral lines. However this kind of analysis would require algorithms which are rather more involved than those used in the present analysis (such algorithms are presently under investigation $[4,5])$. The analysis here is also complicated by the fact that the different spectra have different resolutions and thus different SNRs, for any given signal.

We restricted our search to a source in the GC emitting a signal at constant frequency during the observation time. We have tracked all the local maxima in each spectrum obtained by setting a threshold [5] at $X_{S N R} \simeq 4$. If a spectral line from the GC were present, it should show up in all the spectra (at various SNRs) at the same frequency.

Figure 10 shows (top) the time-frequency plot of the selected maxima and their histogram (bottom). The resulting histogram is flat and hence no evidence of straight horizontal lines is present in the top figure. However, the sensitivity of this analysis is much poorer than the previous method, as almost all the selected peaks (86\%) have amplitude greater than $10^{-22}$.

\section{CONCLUSIONS}

A first analysis of the data obtained with the Explorer detector in 1991 was performed, with the aim of searching for continuous GW. The analysis was limited to the frequency range $921.32-921.38 \mathrm{~Hz}$, which contained the plus resonance of the detector, where sensitivity was highest. Doppler corrections on the GW frequency were made under the assumption that the source was still in the GC without any intrinsic frequency spin down.

No signals were observed with amplitude greater than $\bar{h}$ $=2.9 \times 10^{-24}$, using data collected over 95.7 days, for a source located at $\alpha=17.70 \pm 0.01 \mathrm{~h}$ and $\delta=-29.00$ $\pm 0.05 \mathrm{deg}$, having a spindown parameter $\tau \geqslant 10^{8}$ years (that is $\dot{p} \leqslant 1.7 \times 10^{-19} \mathrm{~s} / \mathrm{s}$ ).
The procedures adopted here can be applied to any assumed position in the sky of a GW source, for a greater frequency range, or even for a frequency correction at the source due to spin down and intrinsic Doppler effects.

\section{ACKNOWLEDGMENTS}

We would like to thank Andrzej Krolak for useful discussions.

\section{APPENDIX A: FREQUENCY RESOLUTION OF THE BASIC FFTS}

We report here on the choice of the spectral resolution for our spectral data base $[17,18]$. For this purpose the use of approximate formulas is well justified.

The formula for the frequency modulation of the signal, in the approximation of circular motion and neglecting the spin-down, is given by [20]

$$
\nu(t)=\nu_{0}-A \sin \left(\Omega_{r o t} t+\phi_{a}-\alpha\right)+B \sin \left(\Omega_{o r b} t+\phi_{b}\right)
$$

where $\Omega_{r o t}$ is the angular sidereal frequency, $\Omega_{\text {orb }}$ the angular orbital frequency, $\phi_{a}, \phi_{b}$ constant phases, $\alpha$ is the right ascension, $\nu_{0}$ the frequency of the GW at the source. The amplitude $A$ of the sidereal period is given by

$$
A=\nu_{0} R_{E} \Omega_{r o t} \cos \phi \cos \delta / c
$$

where $R_{E}$ is the Earth radius, $\phi$ is the latitude of the detector, $c$ is the velocity of the light and $\delta$ is the declination of the source.

The sidereal component produces a maximum time derivative of the frequency given by

$$
a=\left|\frac{d \nu_{\text {rot }}}{d t}\right|_{\text {max }}=A \cdot \Omega_{\text {rot }}=\nu_{0} 11.244 \times 10^{-11} \cos \phi \mathrm{Hz} / \mathrm{s}
$$

where $\nu_{0}$ is measured in $\mathrm{Hz}$.

The amplitude $B$ of the annual modulation is of the order of

$$
B=\nu_{0} R_{o r b} \Omega_{o r b} / c
$$

where $R_{o r b}$ is the radius of the orbit of the Earth around the Sun.

This component produces a maximum time derivative of the frequency given by

$$
b=\left|\frac{d \nu_{\text {orb }}}{d t}\right|_{\text {max }}=B \cdot \Omega_{\text {orb }}=\nu_{0} 1.977 \times 10^{-11} \mathrm{~Hz} / \mathrm{s}
$$

where $\nu_{0}$ is measured in $\mathrm{Hz}$. In order to have a bin width greater than the maximum frequency variation expected for the Doppler effect during $t_{0}$ we must choose the time duration of our basic spectra $t_{0}$ such that

$$
1 / t_{0}>t_{0} \cdot(a+b)
$$


If we consider the Explorer latitude and $\nu_{0}=921.38 \mathrm{~Hz}$ we get

$$
t_{0}<3339 \text { s. }
$$

In our data, the sampling time is $\delta_{t_{f}}=18.176 \mathrm{~ms}$; then, using $t_{0}=2382.35 \mathrm{~s}$, that is $0.6617 \mathrm{~h}$, we have a frequency resolution

$$
\delta \nu=0.41975 \mathrm{mHz}
$$

[while the maximum frequency variation due to the Doppler effect during the time $t_{0}$ is of the order of $t_{0} \cdot(a+b)$ $=0.215 \mathrm{mHz}$ ], that is $2^{17}=131072$ samples in each periodogram.

\section{APPENDIX B: PRACTICAL ISSUES IN THE PROCEDURE FOR COMBINING THE SPECTRA COHERENTLY}

Each FFT is computed using $2 N$ data, sampled with sampling time $\Delta t$. The data are windowed, in the time domain, before the Fourier transform. This means that the data $y_{i}$ are multiplied by the weights $w_{i}=A-B \cos (i)+C \cos (2 i)$, where $i=(0,2 N-1) \cdot 2 \pi /(2 N-1)$. In the present analysis we have used a Hamming window, that is $A=0.54, B=0.46$, and $C$ $=0$.

The FFTs are stored in units of strain $\sqrt{\mathrm{Hz}}$, and are normalized so that their squared modulus is the spectrum.

The basic FFTs of the data base overlap for half their length. The time duration of each FFT is $t_{0}=2 N \Delta t$, and a new FFT is done after time $t_{0} / 2$. This is important since it avoids distortions in the final time domain sequence- this is the well known "overlap-add" method, described in many data analysis textbooks. For example, for the Explorer detector we have 110 overlapped FFTs over 36 hours $(\delta \nu$ $=0.41 \ldots \mathrm{mHz}$.

We select the frequency range to be analyzed and we add zeros to construct the analytical signal. These data should be to a power of 2, to allow use of a fast Fourier algorithm. The chosen frequency range should be wide enough to include all the frequencies we expect to observe due to the Doppler shift from the given source, during the time of observation.

After the bandwidth has been selected, the data (still in the frequency domain) should be windowed, to avoid edge effects in the transformed data.

The selected data are then transformed to return to the time domain. At this stage we must remove the window used in the data when constructing the FFT data base, by simply dividing the new time domain data by the weights $w_{i}$. This operation recovers the original time data (sub-sampled) because the only regions where the division might not work are the edges of the data stream, where the value of $w_{i}$ may be zero, depending on the kind of window used (a problem which, of course, has been overcome by the overlapping of the FFTs).

If an FFT under consideration is vetoed or if it is missing, then the data are set to zero.

Each new group of time domain data is appended to the previous groups, after elimination of the overlapped data. Since the overlapping concerns half the data we eliminate

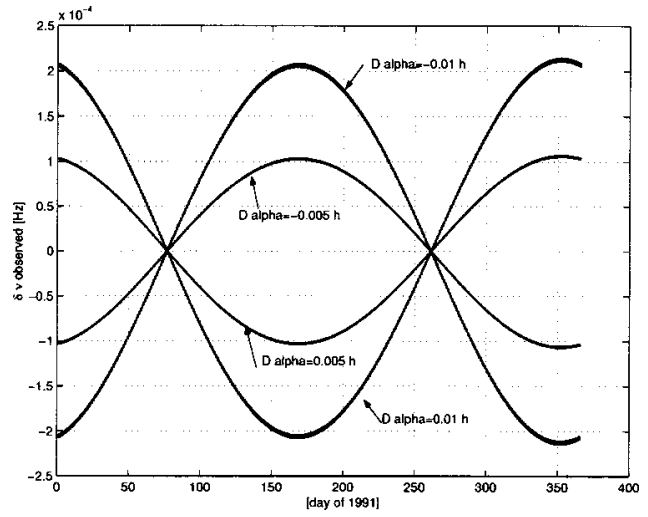

FIG. 11. The graph shows the difference in the observed frequency on Earth between a signal in the GC and signals coming from nearby coordinates. The $x$-axis are days of 1991 . The $y$-axis are the frequencies, from $-2.5 \times 10^{-4}$ to $2.5 \times 10^{-4} \mathrm{~Hz}$.

$1 / 4$ of the data at the beginning and end of each stream. The first 1/4 data in the first FFT and the last 1/4 in the last FFT can be discarded. The data of missing or vetoed periods, set to zero as explained above, are appended to the others in the same way.

At this stage we have a sub-sampled time domain data stream, which represents the analytical signal associated with the original data.

Now we can take into account the Doppler shift and correct the data as previously explained.

The final step is to calculate the power spectrum from this sub-sampled time domain data (after data windowing in the time domain).

\section{APPENDIX C: UNCERTAINTY IN THE SOURCE PARAMETERS}

\section{Uncertainty in the source position parameters}

In this analysis we used the coordinates $\alpha=17.7 \mathrm{~h}, \delta$ $=-29.0 \mathrm{deg}$ to define the GC. In order to calculate the region of the sky effectively covered by this definition it was necessary to study the effect on the analysis of a source not being "exactly" in the GC, since the frequency modulation depends on the precise location of the source. To get an idea of the problem in 1991 we plotted (Fig. 11) the difference in the observed frequencies on Earth between a signal from the GC and signals coming from sources at nearby coordinates.

From the graph it is easy to see that differences $\Delta \alpha$ in the right ascension of $\pm 0.01 \mathrm{~h}$ lead to maximum differences in the observed frequencies $\Delta \nu_{0}$ of $\simeq \pm 2 \times 10^{-4} \mathrm{~Hz}$.

This mismatch is maximum twice a year, at the beginning of June and at the beginning of December. Thus, we studied the effect of the mismatch during a run in December, when it was maximum.

From the figure it is also possible to note that, for the considered (small) differences in the values of right ascension, if $\Delta \nu_{0}$ is the frequency change due to $\Delta \alpha$, the frequency change due to $-\Delta \alpha$ is $-\Delta \nu_{0}$.

This effect can be derived using Eq. (A1) (Appendix A), in the approximation $(1-\cos \Delta \alpha) \simeq 0$. 
TABLE I. An example of the effect due to uncertainties in right ascension and declination.

\begin{tabular}{|c|c|c|c|}
\hline$\Delta \alpha \mathrm{h}$ & $\Delta \delta \operatorname{deg}$ & signal energy & $\Delta f(\mathrm{n}$ bin $)$ \\
\hline 0.000 & -0.30 & $0.25 ; 0.31 ; 0.28$ & +13 \\
\hline 0.000 & -0.20 & $0.24 ; 0.46 ; 0.43$ & +8 \\
\hline 0.000 & -0.10 & $0.35 ; 0.79 ; 0.37$ & +4 \\
\hline 0.000 & -0.05 & $0.40 ; 0.95 ; 0.26$ & +2 \\
\hline 0.000 & 0.000 & $0.46 ; 1.00 ; 0.17$ & +0 \\
\hline 0.000 & +0.05 & $0.51 ; 0.92 ; 0.13$ & -2 \\
\hline 0.000 & +0.10 & $0.56 ; 0.74 ; 0.13$ & -4 \\
\hline 0.000 & +0.20 & $0.31 ; 0.51 ; 0.35$ & -9 \\
\hline 0.000 & +0.30 & $0.24 ; 0.33 ; 0.29$ & -14 \\
\hline 0.005 & -0.30 & $0.26 ; 0.26 ; 0.15$ & +143 \\
\hline 0.005 & -0.20 & $0.35 ; 0.37 ; 0.23$ & +139 \\
\hline 0.005 & -0.10 & $0.31 ; 0.59 ; 0.39$ & +134 \\
\hline 0.005 & -0.05 & $0.40 ; 0.72 ; 0.35$ & +131 \\
\hline 0.005 & 0.000 & $0.47 ; 0.85 ; 0.27$ & +129 \\
\hline 0.005 & +0.05 & $0.50 ; 0.95 ; 0.19$ & +128 \\
\hline 0.005 & +0.10 & $0.50 ; 0.96 ; 0.14$ & +126 \\
\hline 0.005 & +0.20 & $0.51 ; 0.66 ; 0.18$ & +127 \\
\hline 0.005 & +0.30 & $0.29 ; 0.43 ; 0.33$ & +116 \\
\hline 0.008 & -0.30 & $0.21 ; 0.26 ; 0.18$ & +220 \\
\hline 0.008 & -0.20 & $0.24 ; 0.34 ; 0.29$ & +206 \\
\hline 0.008 & -0.10 & $0.43 ; 0.47 ; 0.25$ & +202 \\
\hline 0.008 & -0.05 & $0.19 ; 0.55 ; 0.52$ & +199 \\
\hline 0.008 & 0.000 & $0.18 ; 0.71 ; 0.53$ & +197 \\
\hline 0.008 & +0.05 & $0.15 ; 0.88 ; 0.52$ & +195 \\
\hline 0.008 & +0.10 & $0.13 ; 0.96 ; 0.51$ & +193 \\
\hline 0.008 & +0.20 & $0.23 ; 0.79 ; 0.50$ & +189 \\
\hline 0.008 & +0.30 & $0.34 ; 0.50 ; 0.35$ & +184 \\
\hline 0.010 & -0.30 & $0.16 ; 0.23 ; 0.21$ & +271 \\
\hline 0.010 & -0.20 & $0.26 ; 0.31 ; 0.24$ & +266 \\
\hline 0.010 & -0.10 & $0.24 ; 0.44 ; 0.38$ & +261 \\
\hline 0.010 & -0.05 & $0.29 ; 0.56 ; 0.38$ & +260 \\
\hline 0.010 & 0.000 & $0.35 ; 0.67 ; 0.36$ & +255 \\
\hline 0.010 & +0.05 & $0.42 ; 0.79 ; 0.33$ & +256 \\
\hline 0.010 & +0.10 & $0.43 ; 0.92 ; 0.27$ & +254 \\
\hline 0.010 & +0.20 & $0.36 ; 0.95 ; 0.24$ & +250 \\
\hline 0.010 & +0.30 & $0.40 ; 0.59 ; 0.29$ & +246 \\
\hline 0.020 & -0.30 & $0.11 ; 0.18 ; 0.15$ & +429 \\
\hline 0.020 & -0.20 & $0.21 ; 0.23 ; 0.12$ & +426 \\
\hline 0.020 & -0.10 & $0.23 ; 0.30 ; 0.23$ & +421 \\
\hline 0.020 & -0.05 & $0.29 ; 0.34 ; 0.25$ & +419 \\
\hline 0.020 & 0.000 & $0.35 ; 0.40 ; 0.24$ & +417 \\
\hline 0.020 & +0.05 & $0.43 ; 0.45 ; 0.22$ & +415 \\
\hline 0.020 & +0.10 & $0.16 ; 0.52 ; 0.49$ & +412 \\
\hline 0.020 & +0.20 & $0.08 ; 0.68 ; 0.62$ & +408 \\
\hline 0.020 & +0.30 & $0.64 ; 0.78 ; 0.12$ & +405 \\
\hline
\end{tabular}

Table I shows the results for uncertainties both in right ascension and in declination.

It is hence important to note that we report in the table the results of the simulation only for positive values of $\Delta \alpha$. In fact, as explained before, the resulting frequency variation due to a (small) mismatch $\pm \Delta \alpha$ is symmetric. We must note,

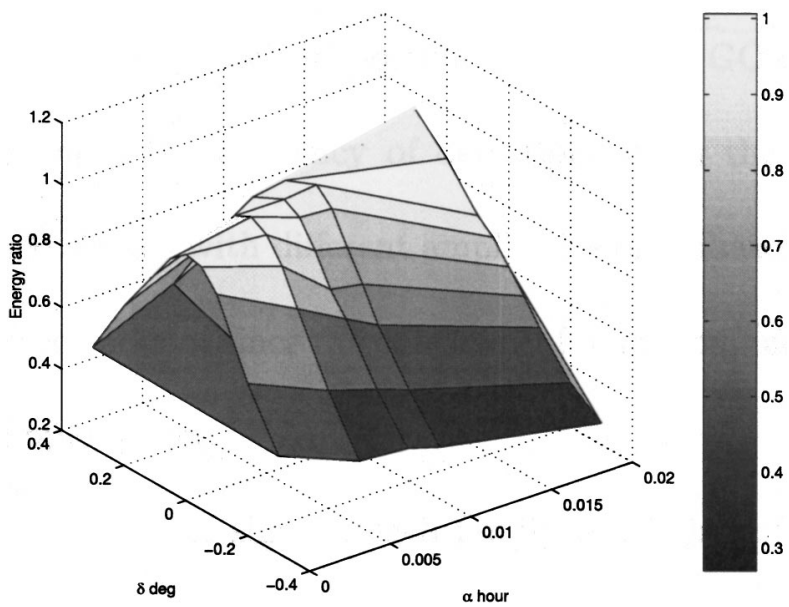

FIG. 12. Three-dimensional plot of the data in Table I. The $z$-axis is the energy of the signal integrated over the three bins (maximum previous, next). The $x$ and $y$-axes are the mismatch in right ascension (hours) and in declination (degrees).

from the table, that, for $\Delta \alpha=0$, the frequency variation is symmetric also for a (small) mismatch of $\pm \Delta \delta$.

Thus, in a first approximation, given a result for a pair $(\Delta \alpha, \Delta \delta)$, the same result, but with the opposite sign for $\Delta \nu_{0}$, will be obtained for the pair $(-\Delta \alpha,-\Delta \delta)$. We have tested this also with a Monte Carlo simulation on a few points from the table.

The first column in the table gives the error in right ascension ( $\Delta \alpha$ in hours); the second, the error in declination ( $\Delta \delta$ in degrees); the third, the energy of the signal, $1 / \mathrm{Hz}$, in the frequency bin of its maximum and in the previous and next bins nearest to the maximum; the fourth column gives the difference in the frequency of the signal compared to the nominal, expressed in number of bins (one bin is 8.1 $\times 10^{-7} \mathrm{~Hz}$ ). Figure 12 is the corresponding 3-dimensional plot. The $z$-axis is the energy of the signal, integrated over the three bins.

It is not easy to arrive at a general conclusion, because the final effect depends very much on the uncertainty on right ascension and declination. In some cases, when the two parameters act in opposite direction, the final result is better compared to a mismatch in only one of the two parameters. This is why, for example, the energy absorbtion when

TABLE II. Results for uncertainty in the source frequency. The first column gives the error in the correction frequency $(\Delta \nu$ in $\mathrm{Hz})$; the second, the energy of the signal, $1 / \mathrm{Hz}$, in the frequency bin of its maximum and in the previous and next bins nearest to the maximum; the third column gives the difference in the frequency of the signal compared to the nominal, expressed in number of bins (one bin is $8.1 \times 10^{-7} \mathrm{~Hz}$ ).

\begin{tabular}{|c|c|c|}
\hline$\Delta \nu$ & signal energy & $\Delta f(\mathrm{n}$ bin $)$ \\
\hline 0.0 & $(0.46) 1.00(0.17)$ & +0 \\
\hline+0.1 & (0.29) $0.92(0.39)$ & +1 \\
\hline-0.1 & $(0.71) 0.73(0.15)$ & -1 \\
\hline
\end{tabular}


$\Delta \alpha=0.02 \mathrm{~h}$ and $\Delta \delta=0.3 \mathrm{deg}$ is 0.78 , larger than the value 0.40 for $\Delta \alpha=0.02 \mathrm{~h}$ and $\Delta \delta=0 \mathrm{deg}$.

Anyway, assuming the analysis is valid even when there is a worsening by a factor of 2 in the energy absorbtion we may conclude that the region of the sky under study is definitely within either the volume $\alpha=17.7 \pm 0.01 \mathrm{~h}$ and $\delta=$ $-29.0 \pm 0.05 \mathrm{deg}(0.01 \mathrm{~h}=0.15 \mathrm{deg})$, or the volume $\alpha$ $=17.7 \pm 0.005 \mathrm{~h}$ and $\delta=-29.0 \pm 0.2 \mathrm{deg}$.

\section{Uncertainty in the source frequency}

To test the extent to which the analysis depends on knowledge of the intrinsic frequency of the source, we did a simu- lation of a spectrum of 14.1 days, by introducing a signal at 921.3 Hz and correcting it, during Doppler removal, using 921.2 and $921.4 \mathrm{~Hz}$ (that is with an error of $\pm 0.1 \mathrm{~Hz}$ ). From Table II it is easy to see that there are no significant differences in the resulting spectra. Thus the final result is only slightly affected by even a very "big" error such as this. This finding is important because it allows us to analyze just a set of discrete frequencies, for example just $1 / 100$ of the frequencies in the original FFTs $(0.419 \ldots \mathrm{mHz})$. It can be shown that this property is intrinsic to the nature of the Doppler correcting factor [Eq. (9)], which depends on the difference between the intrinsic and the observed frequencies [7].
[1] B. F. Schutz, in Proceedings of the 12th Sigrav Meeting (World Scientific, Singapore, 1997).

[2] K. Thorne, in 300 Years of Gravitation, edited by S. W. Hawking and W. Israel (Cambridge University Press, Cambridge, England, 1987).

[3] F. Camilo, D. R. Lorimer, P. Freire, and A. G. Lyne, Astrophys. J. 535, 975 (2000).

[4] P. R. Brady and T. Creighton, Phys. Rev. D 61, 082001 (2000); P. R. Brady, T. Creighton, C. Cutler, and B. F. Schutz, ibid. 57, 2101 (1998).

[5] M. A. Papa, P. Astone, S. Frasca, and B. F. Schutz, Searching for Continuous Waves by Line Identification, in Albert Einstein Institute AEI-057, 1998; and Proceedings of the GWDAW2, Orsay, 1997, edited by M. Davier and P. Hello (Editions Frontières, Gif-sur-Yvette, 1998).

[6] A. Krolak, "Data analysis for continuous GW signals," gr-qc/9903099; P. Jaranowski and A. Krolak, Phys. Rev. D 61, 062001 (2000); 59, 063003 (1999); P. Jaranowski, A. Krolak, and B. F. Schutz, ibid. 58, 063001 (1998); P. Astone, K. Borkowsi, P. Jaranowski, and A. Krolak, "Data analysis of GW signals from spinning neutron stars. IV. An all sky search," gr-qc/0012108.

[7] P. Astone, S. Frasca, and M. A. Papa, "Main features of the proposed short FFT data base and of the analysis procedures that will operate on it;" "Practical aspects of the proposed targeted search with short FFTs" at the "Working group on algorithms for reconstructing data from short FFT data base," Joint meeting, Max Planck Institut of Potsdam and University of Rome "La Sapienza," Rome, 1998 (this and related material are available at http://www.roma1.infn.it/rog/astone and http://grwav1.roma1.infn.it/dadps).

[8] P. Astone, M. Bassan, P. Bonifazi, P. Carelli, M. G. Castellano, G. Cavallari, E. Coccia, C. Cosmelli, V. Fafone, S. Frasca, E. Majorana, I. Modena, G. V. Pallottino, G. Pizzella, P. Rapagnani, F. Ricci, and M. Visco, Phys. Rev. D 47, 362 (1993). From 1998 Explorer is a CERN recognized experiment.

[9] P. Astone, M. Bassan, P. Bonifazi, P. Carelli, E. Coccia, C. Cosmelli, V. Fafone, S. Frasca, K. Geng, W. O. Hamilton, W. W. Johnson, E. Mauceli, M. P. McHugh, S. Merkowitz, I. Modena, P. Modestino, A. Morse, G. V. Pallottino, M. A. Papa, G. Pizzella, N. Solomonson, R. Terenzi, M. Visco, and N. Zhu, Phys. Rev. D 59, 122001 (1999).

[10] P. Astone, M. Bassan, D. G. Blair, P. Bonifazi, P. Carelli, E. Coccia, V. Fafone, L. Febo, S. Frasca, L. S. Heng, E. N.
Ivanov, A. Marini, S. M. Merkowitz, Y. Minenkov, I. Modena, G. Modestino, A. Moleti, G. V. Pallottino, M. A. Papa, G. Pizzella, F. Ronga, R. Terenzi, M. E. Tobar, P. J. Turner, F. J. van Kann, M. Visco, and L. Votano, Astropart. Phys. 10, 83 (1998).

[11] Z. A. Allen, P. Astone, L. Baggio, M. Bassan, D. G. Blair, M. Bonaldi, P. Bonifazi, P. Carelli, M. Cerdonio, E. Coccia, L. Conti, C. Cosmelli, V. Crivelli Visconti, S. D' Antonio, V. Fafone, P. Falferi, P. Fortini, S. Frasca, W. O. Hamilton, L. S. Heng, E. N. Ivanov, W. W. Johnson, C. R. Locke, A. Marini, V. Martinucci, E. Mauceli, M. P. McHugh, R. Mezzena, Y. Minenkov, I. Modena, G. Modestino, A. Moleti, A. Ortolan, G. V. Pallottino, G. Pizzella, G. A. Prodi, E. Rocco, F. Ronga, F. Salemi, G. Santonastasi, L. Taffarello, R. Terenzi, M. E. Tobar, G. Vedovato, A. Vinante, M. Visco, S. Vitale, L. Votano, and J. P. Zendri, Phys. Rev. Lett. 85, 5046 (2000).

[12] P. Astone, C. Buttiglione, S. Frasca, G. V. Pallottino, and G. Pizzella, Nuovo Cimento Soc. Ital. Fis., C 20C, 9 (1997).

[13] G. V. Pallottino and G. Pizzella, Nuovo Cimento Soc. Ital. Fis., C 7C, 155 (1984).

[14] P. Astone, G. V. Pallottino, and G. Pizzella, Class. Quantum Grav. 14, 2019 (1997).

[15] P. Astone, M. Bassan, P. Bonifazi, P. Carelli, E. Coccia, C. Cosmelli, V. Fafone, S. Frasca, S. Marini, G. Mazzitelli, P. Modestino, I. Modena, A. Moleti, G. V. Pallottino, M. A. Papa, G. Pizzella, P. Rapagnani, F. Ricci, F. Ronga, R. Terenzi, M. Visco, and L. Votano, Astropart. Phys. 7, 231 (1997).

[16] G. Prodi, in Initial Operation of the GW Detector Auriga, Proceedings of the Second Edoardo Amaldi Conference on Gravitational Wave Experiments, edited by E. Coccia et al. (World Scientific, Singapore, 1998), pp. 148-158.

[17] J. Livas, Broadband Search Techniques for Periodic Sources of Gravitational Radiation, in Gravitational Wave Data Analysis, edited by B. F. Schutz (Kluwer Academic, Dordrecht, 1989), p. 217.

[18] C. La Posta and S. Frasca, Nuovo Cimento Soc. Ital. Fis., C 14C, 235 (1991).

[19] See, for example, S. A. Tretter, Introduction to Discrete Time Signal Processing (Wiley, New York, 1976).

[20] See Eq. (18) in P. Jaranowski, A. Krolak, and B. F. Schutz, Phys. Rev. D 58, 063001 (1998). Equation (18) of the above paper contains the formula for the phase. Time derivative of it (divided by $2 \pi$ ) gives the instananeous frequency. 